\title{
Familienleben zwischen prekären Arbeitswelten und Konsu- merwartungen: Ein Beitrag zur sozioökonomischen Bildung im GW-Unterricht
}

\author{
martin.duer@ph-vorarlberg.ac.at, Pädagogische Hochschule Vorarlberg \\ ** christian.reiner@univie.ac.at, Universität Wien \\ *** christian.vielhaber@univie.ac.at, Universität Wien
}

eingereicht am: 05.09.2016, akzeptiert am: 09.11.2016

\begin{abstract}
Während die Lebens- und Konsumchancen nach wie vor wesentlich über die Teilnahme am Erwerbsprozess bestimmt werden, sieht sich der Einzelne/die Einzelne mit zunehmend unsicheren, prekären Arbeitsverhältnissen konfrontiert. Unter diesen Bedingungen erweist sich ein einmal erreichter Lebensstandard als fragiler temporärer Status und der gesellschaftliche Abstieg aufgrund von Arbeitslosigkeit und anschließender prekärer Beschäftigung als reale Möglichkeit. Die damit einhergehenden Problemlagen wirken zurück auf das soziale Gefüge der Familien- bzw. Haushaltsebene. Der vorgestellte Unterrichtsentwurf unternimmt den Versuch, sich dieser komplexen Problemlage aus der Perspektive von Jugendlichen zu nähern und ihnen damit die Möglichkeit zu geben, ihren eigenen (zukünftigen) sozioökomischen Status auf Arbeits- und Konsumgütermärkten sowie die Wechselwirkungen mit ihren familiären Verhältnissen zu reflektieren.
\end{abstract}

Keywords: Lebensstandard, Familienleben, prekäre Beschäftigung, sozioökonomische Bildung, Handlungsorientierung

\section{Family life between non-standard work and conspicuous consumption: A contribution to socio- economic education}

The prospect for a decent standard of living still depends to a large extent on participation in the labour market. Yet workers and job seekers are confronted with a labour market that offers increasingly insecure, precarious jobs. Under such circumstances, a living standard, once achieved, cannot be taken for granted, since unemployment and low-quality jobs befall ever more workers. Moreover, labour market problems are not without repercussions for the social relations of workers in general and their family life in particular. The proposed design of a teaching unit enables pupils to reflect upon those socio-economic dynamics from their own perspective.

Keywords: standard of living, family live, non-standard work, socio-economic education, action orientation

\section{$1 \quad$ Einleitung}

„... einen tollen Beruf (...) mit entsprechendem Gehalt zu bekommen und sich dann auch einen gewissen Luxus (eigenes Auto etc.) leisten zu können".

„... genug Geld zur Verfügung haben, nicht an der Armutsgrenze zu leben, ein schönes Haus oder eine schöne Wohnung, sich auch Wünsche erfüllen zu können".
Diese zwei Zitate aus einer in Vorarlberg durchgeführten Befragung von 202 Jugendlichen über ihre Vorstellungen von Lebensqualität zeigen die hohe Bedeutung materieller Güter für Jugendliche in unserer Region. $62 \%$ der befragten Jugendlichen nennen in einem durchgeführten Freewriting ${ }^{2}$ zum The-

\footnotetext{
In einem Freewriting werden innerhalb einer kurzen vorgegebenen Zeit die persönlichen Gedanken zu einem bestimmten Thema niedergeschrieben, ohne dabei sprachliche oder inhaltliche Regeln zu berücksichtigen. Durch einen durchgehenden Schreibfluss sollen alle Gedanken zu einem vorgegebenen Thema unmittelbar zu Papier gebracht werden (Elbow 1998).
} 
ma Lebensqualität explizit Luxusgüter, die sie für ihr persönliches Wohlbefinden als wichtig erachten (Dür 2016). Diese Ergebnisse stehen im Einklang mit der Studie von Haug und Gschwandtner (2006), die die Zukunftserwartungen von Jugendlichen (13-18 jährige Schüler/innen) aus Deutschland und Österreich auf Basis von 500 Aufsätzen qualitativ untersuchten. Die Autorinnen zeichnen ein bemerkenswertes Bild einer materialistisch, auf Konsum und Besitz fixierten Jugend: „Wesentlich (...) ist der Besitz. Dieser konzentriert auch die Entwürfe von Zukunft ins Private, zieht die Energie von der Welt und fixiert auf Konsum." (Haug \& Gschwandtner 2006, 153).

\section{Fachdidaktische Relevanz und Lehrplan- bezug}

Wenngleich eine derartige Orientierung an sich bereits als problematisch identifiziert werden könnte (etwa aufgrund der Notwendigkeit von Klimaschutzmaßnahmen auf individueller und kollektiver Ebene, die eher eine Abkehr von einer stark materialistisch ausgerichteten Lebensweise voraussetzen würden), ist sie potenziell auch im Widerspruch zu aktuell vorhandenen und künftig zu erwartenden Budgetausstattungen der Schüler/innen. Insbesondere entsteht eine zunehmende Kluft zwischen der realen Einkommensentwicklung, sowie dem verfügbaren Vermögen und den steigenden Konsumwünschen der Jugendlichen. Wer kein Vermögen erbt, wird auch in Zukunft von einem Arbeitseinkommen abhängig sein. Hier ergibt sich aber das Problem, dass der Arbeitsmarkt nicht mehr die gleiche Berechenbarkeit bietet wie noch vor einigen Jahrzehnten. Prekäre Beschäftigungsverhältnisse sorgen dafür, dass niedrige Einkommen und Unsicherheit geplante Lebensentwürfe verunmöglichen und möglicherweise in ihr Gegenteil verkehren können. Die Thematik ist nicht zuletzt vor dem Hintergrund zu betrachten, dass junge Menschen deutlich überproportional von prekärer Beschäftigung betroffen sind (Teitzer 2014). Nicht zuletzt ist der Berufseinstieg für junge Menschen in bestimmten Branchen (wie z.B. in der Kreativwirtschaft) an die Akzeptanz unbezahlter Praktika gebunden („Generation Praktikum"). ${ }^{3}$ Im Zusammenhang mit prekärer Beschäftigung ist freilich auch das Thema Jugendarbeitslosigkeit zu erwähnen. Diese ist zwar in Österreich deutlich niedriger als im EU-Durchschnitt aber ebenfalls höher als die Arbeitslosenquote insgesamt (EuroMemorandum 2016).

Siehe z. B. http://derstandard.at/2000013971813/GenerationPraktikum-macht-nach-Abschluss-kaum-Praktika (06.08.2016).
Deshalb erscheint es notwendig, die Problematik prekärer Arbeitsverhältnisse und die daraus resultierenden Widersprüche im GW-Unterricht zu thematisieren und die Schüler/innen in die Lage zu versetzen, eigene Lebensentwürfe vor dem Hintergrund ökonomischer Realitäten $\mathrm{zu}$ bewerten und gegebenenfalls in Frage zu stellen (Vielhaber 2001). In diesem Sinne soll die nachstehend vorgestellte Unterrichtssequenz eine Anregung zur Aufklärung und kritischen Reflexion sein, wobei die Schlussfolgerungen im Sinne individueller Bewältigungsstrategien der Ausgangspunkt für gesellschafts- und wirtschaftspolitische Alternativen sein können und sollen. Damit positioniert sich der dargestellte Entwurf bewusst als Beitrag zur sozioökonomischen Bildung im Sinne von Hedkte (2015) und damit gleichzeitig im Gegensatz zu einer verkürzten ökonomistischen Bildung. Letztere zeichnet sich vor allem dadurch aus, dass der Gegenstandsbereich „Wirtschaft“ isoliert, d.h.unter Ausblendung von Wechselwirkungen mit der sozialen und politischen Realität, konzeptualisiert und präsentiert wird.

Im Folgenden wird zunächst kurz auf den Begriffsinhalt und die empirische Bedeutung von prekärer Beschäftigung eingegangen. Anschließend wird eine Unterrichtssequenz vorgestellt, im Zuge derer sich die Schüler/innen mit den möglichen Konsequenzen eines sinkenden Familieneinkommens auseinandersetzen. Das auslösende Moment hierzu ist der Wechsel von einem regulären Beschäftigungsverhältnis hin zu einem atypischen Job. Dabei werden die Schüler/innen aufgefordert, ihre eigenen Strategien im Umgang mit dieser Situation zu erarbeiten. Vorschläge für Arbeitsblätter und Materialien runden den Beitrag ab.

In der AHS Oberstufe bietet das Basiskonzept „Arbeit, Produktion und Konsum“ sowie die Lehrplanbereiche „Menschen und ihre wirtschaftlichen Bedürfnisse“ in der 5. Klasse oder „Gesamtwirtschaftliche Leistungen und Probleme - Wirtschafts- und Sozialpolitik" in der 7. Klasse (BMBF 2004) geeignete Anknüpfungspunkte, um die Schüler/innen mit dem Themenbereich „prekäre Arbeitsverhältnisse“ zu konfrontieren. Auch für die 3. Klasse Unterstufe kann diese Unterrichtssequenz in einer entsprechenden altersadäquaten Modifikation einen geeigneten Einstieg in die angesprochene Problemstellung darstellen. Im Zusammenhang mit dem Thema „Wirtschaften im privaten Haushalt" wird im für diese Schulstufe gültigen Lehrplan als ein Lernziel das „Erkennen der Notwendigkeit, im privaten Haushalt Ausgaben den finanziellen Möglichkeiten entsprechend zu planen" (BMBF 2000) formuliert. Das Unterrichtsprinzip "Wirtschafts- und Verbraucher/innenbildung" bietet ebenfalls Anknüpfungspunkte im Inhaltsfeld „Arbeit, Arbeitsmarkt, Beschäftigung, Einkommensverteilung 


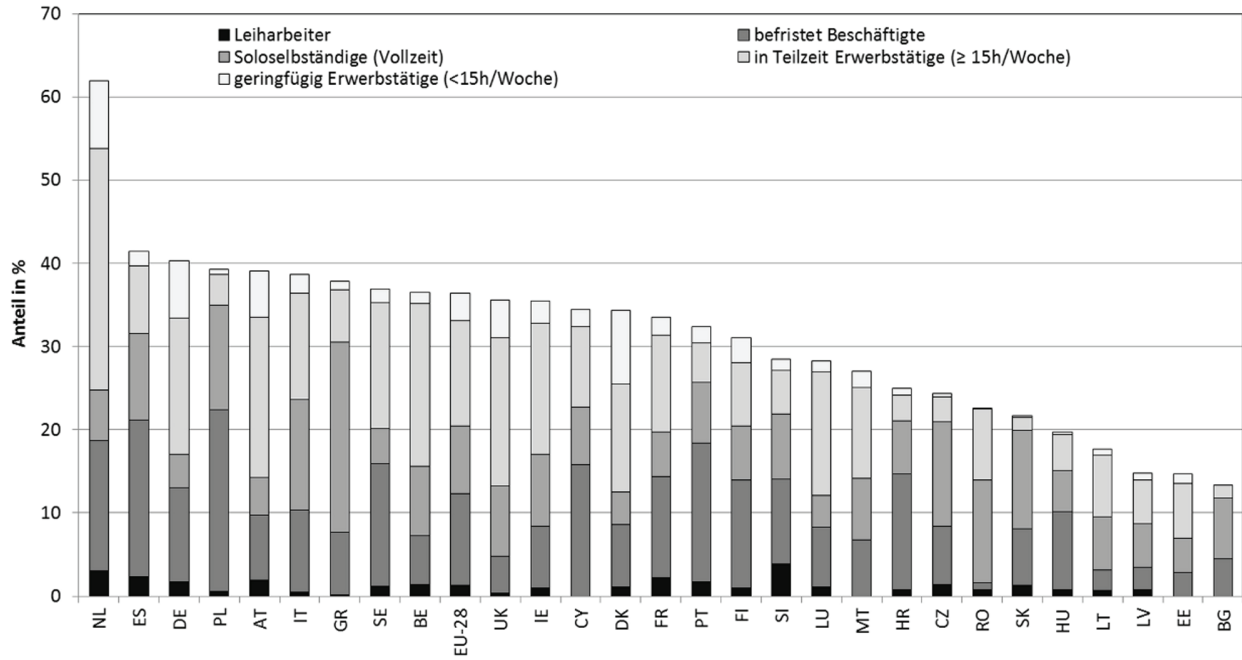

Abb. 1: Anteil atypischer Erwerbstätiger an allen Erwerbstätigen (15-64 Jahre), 2014 (Quelle: eigene Darstellung aus Daten von: Schulze Buschoff 2016, 53. Vgl. auch Schulze Buschoff 2016, 12). sowie deren Zusammenhang mit Bildungsabschlüssen im 21. Jahrhundert" (BMBF 2015, 8).

\section{Prekäre Beschäftigung: Begriff und quan- titative Bedeutung}

Der Begriff der atypischen Beschäftigung bezieht sich in der Regel auf Arbeitsverhältnisse, die relativ zu einem „Normalarbeitsverhältnis“ eine andere Qualität hinsichtlich verschiedener Dimensionen (wie z.B. Lohn, Leistungsdruck, Kündigungsschutz, Flexibilitätserfordernisse) aufweisen (Bosch 2012, Weinkopf et al. 2009). Als Normalarbeitsverhältnis wird dabei üblicherweise eine kollektivvertraglich erfasste, unbefristete Vollzeitbeschäftigung verstanden. Der Begriff der prekären Beschäftigung umfasst jene atypischen Beschäftigungsverhältnisse, die eine geringere Jobqualität als ein Normalarbeitsverhältnis aufweisen und daher negative Effekte auf die Lebenslagen von Menschen haben können. Es gehört zu den gesellschaftspolitisch problematischen Dimensionen von prekärer Beschäftigung, dass diese tendenziell durch (1) geringere Bezahlung und/oder geringeres Lohnwachstum (2) sowie durch ein erhöhtes Risiko gekennzeichnet sind. Das Risiko steigt beispielsweise durch ein relativ geringeres Ausmaß an sozialer Absicherung, wenn der Kündigungsschutz gelockert wird oder aufgrund einer Befristung erst gar keiner besteht oder wenn unternehmerisches Risiko auf abhängig Beschäftigte überwälzt wird. Auf makroökonomischer Ebene gilt die Zunahme von atypischer Beschäftigung als eine der Ursachen für die simultan steigende Einkommensungleichheit in den OECD-Staaten (OECD 2015).

Es sollte nicht unerwähnt bleiben, dass der Begriff der „prekären Beschäftigung" nicht unumstritten ist, und zwar vor allem deswegen, weil atypische Beschäftigung nicht automatisch nachteilig für jeden
Haushalt zu beurteilen ist. Das Institut für Arbeitsmarkt- und Berufsforschung versteht unter dem Begriff „Prekäre Beschäftigung“ Arbeitsverhältnisse mit niedrigen Löhnen, die häufig nicht auf Dauer und Kontinuität angelegt sind, keine Absicherung durch die Sozialversicherung und nur geringe arbeitsrechtliche Schutzrechte aufweisen. "4 Nachdem der vorliegende Beitrag die problematische Seite von atypischer Beschäftigung im Fokus hat, verwenden wir in weiterer Folge den Begriff der prekären sowie den der atypischen Beschäftigung synonym.

Die quantitative Erfassung von atypischer Beschäftigung erfolgt zumeist über die Zuordnung von unterschiedlichen Arbeitsvertragstypen zu typischer oder atypischer Beschäftigung. In empirischen Studien werden dabei unter anderem folgende Kategorien von atypischer Beschäftigung unterschieden: befristet Beschäftige, Teilzeit oder geringfügig Erwerbstätige, Soloselbständige, und Leiharbeiter/innen (vgl. Abb. 1). In Europa ist mehr als ein Drittel aller Erwerbstätigen von atypischer Beschäftigung betroffen (Schulze Buschoff 2016). Österreich weist gemäß Abb. 1 mit ca. $40 \%$ einen etwas überdurchschnittlichen Anteil auf, allerdings ist hierfür v.a. der hohe Anteil an Teilzeitbeschäftigung verantwortlich. Im internationalen Vergleich wird aber auch deutlich, dass die durchschnittliche Jobqualität in Österreich relativ gut ist (Scarpetta et al. 2016).

Im Zeitverlauf zeigt sich für zahlreiche OECDund EU-Staaten eine trendhafte Zunahme an prekärer Beschäftigung. Abbildung 2 zeigt diese Entwicklung auf Basis der neu geschaffenen bzw. vernichteten Arbeitsplätze im Zeitraum 2007-2013. Demnach sind in den OECD Staaten die typischen Beschäftigungs-

http://infosys.iab.de/infoplattform/dokSelect. asp?pkyDokSelect=27\&show=Lit (21.07.2016). Siehe auch die dort zitierte Literatur zum Thema. 


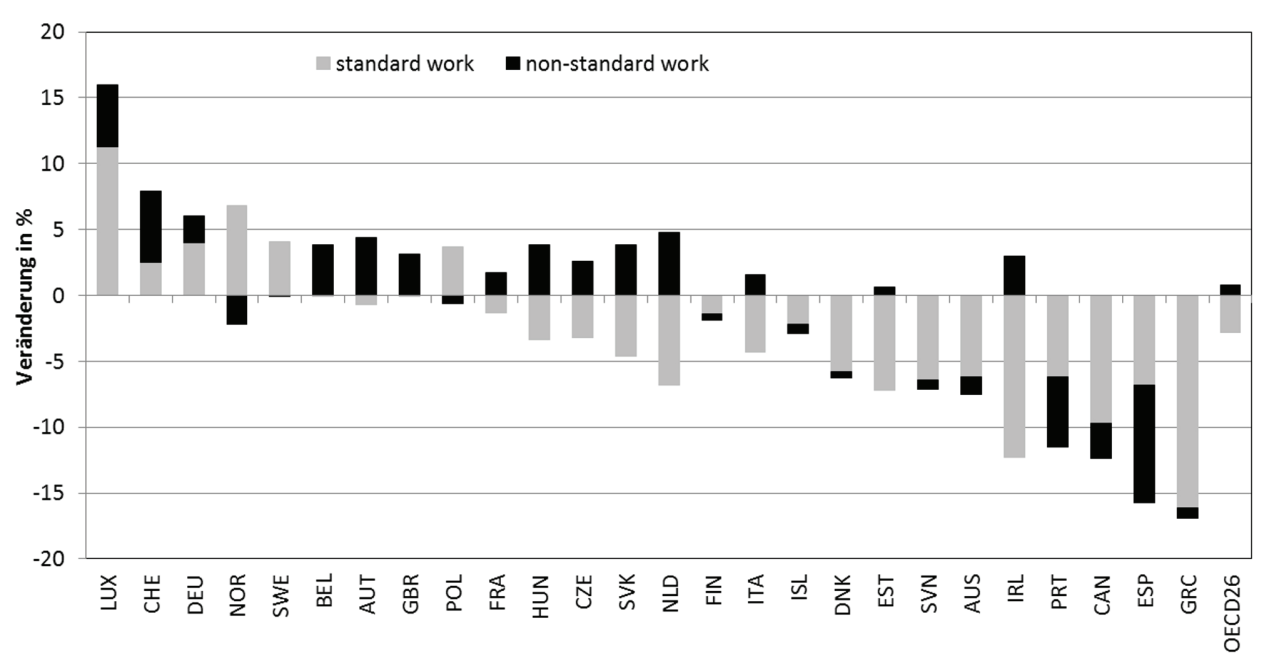

Abb. 2: Beschäftigungsentwicklung von 2007-2013 in Prozent, differenziert nach typischer (standard work) und atypischer Beschäftigung (non-standard work). (Quelle: Eigene Abbildung nach Daten von: OECD 2015). verhältnisse rückläufig, wogegen die Zahl der atypischen Beschäftigungsverhältnisse leicht ansteigt. Österreich (AUT) zählt zu jenen Staaten, in denen die atypischen Beschäftigungsverhältnisse besonders stark zugenommen haben. Der steigende Trend zur atypischen Beschäftigung wird z. B.auch in Allmendinger et al. (2012) oder in Teitzer (2014) bestätigt und diskutiert.

Die soeben aufgezeigten Veränderungen der Beschäftigungsverhältnisse, bzw. die sich daraus ergebenden Folgen, werden damit in Zukunft verstärkt auch die heutigen Schüler/innen betreffen. Der GWUnterricht ist deshalb gefordert, sich beim Thema Arbeitsmarkt nicht nur auf die Behandlung klassischer Normarbeitsverhältnisse zu beschränken, sondern vermehrt auch diese neuen Prozesse und deren mögliche zukünftige Auswirkungen zu thematisieren. Das im Folgenden ausführlich dargelegte Unterrichtskonzept soll diesen neuen Entwicklungen Rechnung tragen und Schüler/innen dazu anleiten, sich auf einer persönlichen Ebene mit dieser Thematik auseinanderzusetzen und konkrete individuelle Handlungsstrategien auszuarbeiten.

\section{Umsetzung im Unterricht}

Als Einstieg in das Thema werden die Schüler/innen aufgefordert, in Einzelarbeit über ihre Ansprüche an eine rundum zufriedenstellende Lebenswelt nachzudenken und ihre Gedanken in einer besonderen Form sichtbar zu machen. Welche Methode wird dafür gewählt? Es ist die Gestaltung eines persönlichen "Glücksbaumes“, der als Repräsentationsfigur ihrer Bewusstseinsorientierungen dienen soll. Um ihn abbilden zu können, setzen sich die Schüler/innen deshalb zu Beginn der Unterrichtseinheit mit ihren eigenen Vorstellungen von Wohlbefinden auseinander. Die, für das individuelle Wohlbefinden als wichtig erachteten Aspekte, bilden dabei die Blätter dieses Baumes (Arbeitsblatt 1, vgl. Abb.3).

In der Folge sollen die Ergebnisse verglichen und diskutiert werden. Ein erster Schritt könnte als Partnerarbeit inszeniert werden. Die Banknachbarinnen und Banknachbarn setzen sich mit den Wohlfühlfaktoren des jeweils anderen auseinander und halten Ähnlichkeiten sowie Unterschiede in einer Auflistung fest. Im Anschluss daran kann durch die Zusammenführung der Einzelergebnisse auch das Klassenergebnis an der Tafel dargestellt und einer Analyse zugeführt werden. Dabei geht es um die Herausarbeitung von dominanten Wohlfühlfaktoren, die von vielen Schülerinnen Schülern geteilt werden. Ebenso soll aber auch das Spektrum aller genannten Faktoren Beachtung finden, um deutlich zu machen, dass trotz aller gesellschaftlicher Normierungen und peer-group geleiteter Gemeinsamkeiten auch subjektive Aspekte für die persönliche Wohlfühlqualität eine große Rolle spielen können.

Im Folgenden werden die Schüler/innen aufgefordert, sich - erneut in Einzelarbeit - zu jedem der von ihnen angeführten Wohlfühlaspekte mindestens ein konkretes Ereignis zu überlegen, das sich negativ auf diesen Aspekt auswirken könnte. Diese möglichen und von den Schülerinnen und Schülern für sich als relevant erachteten „Störfaktoren“ werden von ihnen ebenfalls in die eigene Zeichnung eingetragen und auf jenem „Baumblatt“ positioniert, auf dem der spezifische, durch die Störung möglicherweise betroffene Wohlfühlfaktor ausgewiesen ist (Arbeitsblatt 2).

Ähnlich wie nach der ersten Diskussionsrunde, bietet sich nach Ende der Eintragungen erneut eine Partnerarbeit zwischen den Banknachbarinnen und Banknachbarn an, wobei der Vergleich der Störfaktoren, die als negative Einflusskräfte auf einen Wohlfühlaspekt wirken, insbesondere dort interessant erscheinen, wo Unterschiede bei den subjektiv diagnostizierten Störfaktoren auftreten, obwohl sie den 


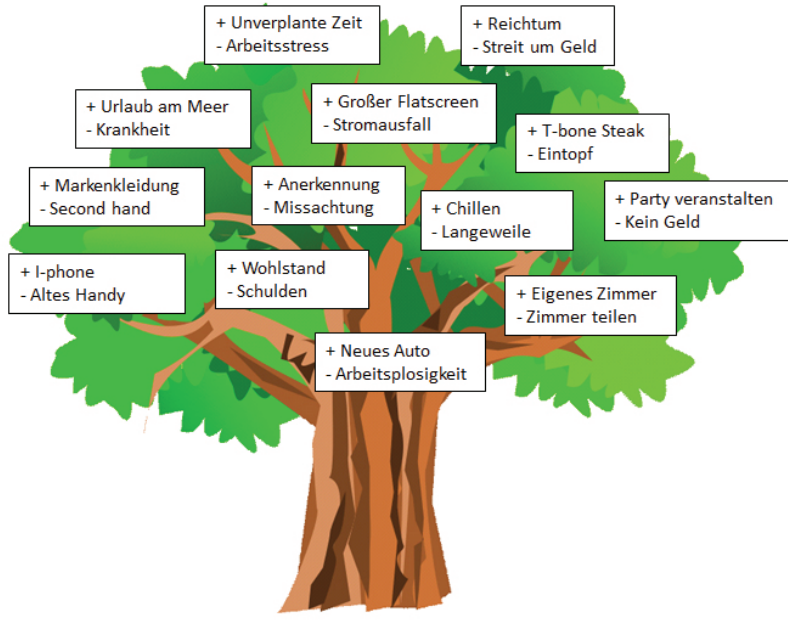

Abb. 3: Beispiel eines Glücksbaumes mit „Woblfühlfaktoren“ (+) und „Störfaktoren“ (-) (eigene Darstellung).

gleichen Wohlfühlfaktor betreffen. Die Selektivität subjektiver Einschätzung wird dabei besonders deutlich und kann zu interessanten Diskussionen führen, wenn es darum geht, die Ursachen unterschiedlicher Prioritätensetzung herauszufinden. Die auftretenden Paarbildungen von Wohlfühl- und Störfaktoren können auch ein fruchtbarer Ansatz sein, um Kausalitäten auf die Spur zu kommen. Die Einzelergebnisse können im Anschluss daran wiederum in das Klassenplenum übergeführt werden.

Den Schülerinnen und Schülern wird in einem nächsten Schritt anhand einer fiktiven Familien- bzw. Einkommenssituation (z. B. zwei Elternteile, beide in einem Beschäftigungsverhältnis und mit einem monatlichen Nettofamilieneinkommen von $€ 3400$, Vater: $€ 2000$, Mutter: $€ 1400)$ eine mögliche Struktur der Haushaltsausgaben vorgestellt (Material 1, Arbeitsblatt 3). Die einzelnen Bereiche, die vom Haushaltsbudget bedient werden müssen, können auch vom Klassenverband erarbeitet werden. In Tabelle 1 ist allerdings bereits ein Vorschlag enthalten, welche "Töpfe“ im Rahmen des Haushaltsbudgets zu berücksichtigen wären. Die Töpfe könnten dann - vom monatlichen Einkommen ausgehend - entweder nach einer entsprechenden Diskussion im Klassenverband oder im Rahmen eines arbeitsgleichen Gruppenunterrichts "gefüllt" werden, wobei das Kriterium der Wirklichkeitsnähe durchaus eine Rolle spielen sollte.

Anschließend wird eine abrupte Änderung der Familieneinkommenssituation angenommen: Der Vater verliert die gut bezahlte Stelle als Facharbeiter und sieht sich nach einer Phase der Arbeitslosigkeit als Leiharbeiter („Zeitarbeitskraft“) mit einem prekären Beschäftigungsverhältnis konfrontiert (Material 1,
Arbeitsblatt 4). ${ }^{5}$ Die damit einhergehende objektive Verschlechterung der Einkommens- und Beschäftigungssituation beeinflusst aber auch die meisten anderen Lebensbereiche negativ. Um diesen Umstand den Schülerinnen und Schülern deutlich zu machen, werden sie aufgefordert, sich konkrete Einsparungsmöglichkeiten zu überlegen, um mit dem verringerten Haushaltseinkommen von $€ 2400$ das Auslangen zu finden. Dieses Einkommen liegt unter der Armutsgefährdungsschwelle (weniger als $60 \%$ des Medianeinkommens) für diesen Haushaltstyp (siehe Material 2).

Die veränderte Einkommenssituation bedeutet, dass überlegt werden muss, wie und mit welchen Konsequenzen die geringeren Budgetmittel nunmehr auf die einzelnen "Töpfe“ verteilt werden könnten. Dabei genügt es nicht, z. B.die Ausgaben für den Bereich Freizeit um eine bestimmte Summe zu verringern, sondern die Schüler/innen werden aufgefordert, konkrete Vorschläge zu machen, wie diese Einsparungen auch tatsächlich erreicht werden können. D.h. für eine Reduktion der Freizeitausgaben ist z. B.ein Verzicht auf das wöchentliche Tennistraining notwendig. Tabelle 1 gibt Beispiele für mögliche Folgen einer Einsparung in den verschiedenen Bereichen an. So könnte beispielsweise an der Peripherie eine billigere Wohnung gefunden werden, aber der Schulweg der Kinder, sowie der Weg zur Arbeit der Eltern würde sich verlängern und die Kosten für Mobilität würden steigen. Schon diese wenigen Beispiele zeigen, dass Schülerinnen und Schülern eindrucksvoll die konkreten Konsequenzen prekärer Arbeitssituationen vor Augen geführt werden können, wenn sie aufgefordert werden, die direkten Auswirkungen auf ihre persönlichen Lebenswelten mitzudenken.

Abschließend zu dieser Lernphase noch ein kurzer Hinweis zu Material 2. Es dient nicht nur der Beförderung des schulischen Lernprozesses, sondern gibt den Schülerinnen und Schülern durch die Ausdifferenzierung in unterschiedliche Haushaltstypen zudem die Möglichkeit auch ihre persönliche Lebenssituation im Hinblick auf Armutsgefährdung abschätzen zu können, ohne diese gegenüber den Mitschülerinnen und Mitschülern offen legen zu müssen - ein zusätzlicher Lernaspekt, der aber wohl nur von Schülerinnen und Schülern der Sekundarstufe 2 als solcher wahrgenommen werden wird. Schüler/innen der 3. Klasse Unterstufe sind mit Überlegungen im Zusammenhang mit der als Beispiel angebotenen Einkommensreduktion und ihren potentiellen Folgen genug gefordert.

Eine Diskussion des Leiharbeiterverhältnisses findet sich z. B. in Schindler \& Schönbauer (2013), Riesenfelder \& Wetzel (2010) sowie Voss \& Vitols (2013). 
Tab.1: Beispiel für eine von Schülerinnen und Schülern überarbeitete Struktur der Haushaltsausgaben

\begin{tabular}{|c|c|c|c|}
\hline Töpfe fürs Haushaltsbudget & $\begin{array}{l}\text { Monatliches Budget: } \\
€ 3400\end{array}$ & $\begin{array}{l}\text { Monatliches } \\
\text { Budget-reduziert: } \\
€ 2400\end{array}$ & $\begin{array}{l}\text { Mögliche Konsequenzen } \\
\text { der Reduktion }\end{array}$ \\
\hline $\begin{array}{l}\text { Wohnen (Miete, Betriebskosten, } \\
\text { Haushaltsgeräte etc.) }\end{array}$ & 800 & 800 & Krisensituation: Schadensfälle \\
\hline $\begin{array}{l}\text { Konsum (Ernährung, Kleidung } \\
\text { etc.) }\end{array}$ & 700 & 500 & Sozialmarkteinkauf \\
\hline $\begin{array}{l}\text { Gesundheit (Medikamente, } \\
\text { Selbstbehalte, etc.) }\end{array}$ & 200 & 100 & Wahlarzt keine Option \\
\hline $\begin{array}{l}\text { Mobilität (Auto, öffentlicher } \\
\text { Verkehr etc.) }\end{array}$ & 350 & 300 & No more taxi \\
\hline $\begin{array}{l}\text { Freizeit (Sportvereine, Ausflüge, } \\
\text { Urlaub etc.) }\end{array}$ & 200 & 100 & $\begin{array}{l}\text { Stornierung der Mitgliedschaft im } \\
\text { Sportclub }\end{array}$ \\
\hline $\begin{array}{l}\text { Versicherungen (Haushalts-, } \\
\text { Pensions-, Rechtsschutzversiche- } \\
\text { rung etc.) }\end{array}$ & 200 & 150 & $\begin{array}{l}\text { Kündigung der Haushaltsversiche- } \\
\text { rung mit möglichen fatalen Folgen }\end{array}$ \\
\hline $\begin{array}{l}\text { Kultur/Bildung (Theaterbesuche, } \\
\text { Ausgaben für Schule etc.) }\end{array}$ & 150 & 50 & $\begin{array}{l}\text { Stornierung des Theaterabonne- } \\
\text { ments }\end{array}$ \\
\hline Sparen/Kreditrückzahlungen & 500 & 250 & Kein Sparauftrag mehr \\
\hline $\begin{array}{l}\text { Sonstiges (Spenden, Taschen- } \\
\text { geld etc.) }\end{array}$ & 300 & 150 & $\begin{array}{l}\text { Keine Spenden mehr, weniger } \\
\text { Taschengeld }\end{array}$ \\
\hline
\end{tabular}

\section{Ausblick und weitere Fragen}

Die Unterrichtssequenz basiert auf der Fallstudie einer einzelnen Familie und verfolgt das Ziel, Schüler/ innen Strategien entwickeln zu lassen, mit denen diese fiktive Familie die sozioökonomische Krise bewältigen könnte. Dies ermöglicht einerseits eine lebensweltnahe Diskussion und eine Reflexion unmittelbar verfügbarer Handlungsparameter, andererseits besteht die Gefahr, dass die strukturellen Ursachen der familiären Problemlage in den Hintergrund treten. Daher wäre es im Sinne der politischen Bildung sowie einer Einbindung volkswirtschaftlicher Bedingungen und Erkenntnisse sinnvoll, die individuelle Familiengeschichte als Ausgangspunkt für allgemeinere Fragen zu nutzen. Beispielhaft seien hier folgende Fragestellungen erwähnt:

1. Warum gibt es überhaupt prekäre Beschäftigungsverhältnisse? Welche Menschen und Branchen sind besonders davon betroffen? Warum haben prekäre Beschäftigungsverhältnisse in den letzten Jahrzehnten zugenommen?

2. Wie werden prekäre Beschäftigungsverhältnisse von den unterschiedlichen Interessenvertretungen bzw. politischen Parteien beurteilt?

3. Welche Auswirkungen auf die gesellschaftliche und wirtschaftliche Entwicklung einer Volkswirtschaft kann die Zunahme prekärer Beschäftigung haben?
4. Welcher Zusammenhang besteht zwischen prekärer Beschäftigung und der Arbeitslosenquote? Sind eine weitere Flexibilisierung und damit auch eine Ausdehnung prekärer Beschäftigungsverhältnisse unvermeidbar, um die Arbeitslosenquote zu senken?

5. Unter welchen Umständen sind prekäre bzw. atypische Beschäftigungsverhältnisse vorteilhaft für eine/n Unternehmer/in, eine/n Arbeitnehmer/in bzw. für eine Familie?

6. Worin liegen die Unterschiede zwischen Lebensstandard und Lebensqualität? Welche Auswirkungen könnten sich durch das Bewusstmachen dieser Differenzen für die persönliche Lebensgestaltung ergeben?

Insbesondere die Fragen 1, 3 und 6 und in entsprechender Modifikation auch die Frage 5 können von den Lehrenden unserer Ansicht nach auch problemlos für Schüler/innen der 3. Klasse Unterstufe altersadäquat adaptiert werden, weil sie auf Problembereiche zielen, die zweifelsohne schon im aktiven Wahrnehmungsbereich dieser Altersstufe liegen.

Der hier vorgelegte Unterrichtsentwurf zum Thema prekäre Beschäftigung versucht eine Annäherung an ein volkswirtschaftlich relevantes und gesellschaftspolitisch kontroverses Thema in lebensnaher und gleichzeitig reflektierter Form. Ziel ist die Aufklärung über soziale Verhältnisse am Arbeitsmarkt, die über vielfa- 
che Mechanismen das Leben von Frauen und Männer und Familien beeinflussen. Schüler/innen mit diesen zum Teil gegensätzlichen Bedingungen zu konfrontieren, trägt dazu bei, ihren Blick auf die Welt und ihre Widersprüche zu schärfen. Damit versucht dieser Beitrag dem Anspruch einer umfassenden sozioökonomischen Bildung im Sinne von Hedke (2015) gerecht zu werden.

Perspektivisch könnte die Auseinandersetzung mit prekären Beschäftigungsverhältnissen dazu beitragen:

1. dass die Jugendlichen für ihre Zukunft verstärkt Strategien verfolgen, die auf eine persönliche $\mathrm{Ri}-$ sikominimierung für prekäre Arbeitsbedingungen abzielen (Ausbildungsweg, Berufswahl etc.);

2. dass in einem Nachdenkprozess die hohe Bedeutung materieller Wünsche kritisch hinterfragt wird und

3. dass eine bewusstere Wahrnehmung von unterschiedlichen Interessenslagen der mehr oder weniger machtvollen Akteure am Arbeitsmarkt bzw. in der Arbeitsmarktpolitik erfolgt.

\section{Literatur}

Allmendinger, J., J. Giesecke, L .Hipp, K. Leuze. \& S. Stuth (2012): Mehr Jobs oder nur schlechte Arbeit? Die Entwicklung atypischer Beschäftigung in Europa. WZBrief Arbeit 13. http://nbn-resolving.de/ urn:nbn:de:101:1-2012082011595 (10.11.2016).

$\mathrm{BMBF}$ - Bundesministerin für Bildung, Wissenschaft und Kultur (2000): Lehrplan für Geographie und Wirtschaftskunde an der AHS Unterstufe. Wien.

BMBF - Bundesministerin für Bildung, Wissenschaft und Kultur (2004): Lehrplan für Geographie und Wirtschaftskunde an der AHS Oberstufe. Wien.

$\mathrm{BMBF}$ - Bundesministerin für Bildung, Wissenschaft und Kultur (2015): Grundsatzerlass zum Unterrichtsprinzip Wirtschafts- und Verbraucher/innenbildung. Wien.

Bosch, G. (2012): Prekäre Beschäftigung und Neuordnung am Arbeitsmarkt. Expertise im Auftrag der Industriegewerkschaft Metall. In: IAQ-Standpunkt. Aktuelle Stellungnahmen aus dem Institut Arbeit und Qualifikation 02. Duisburg.

Dür, M. (2016): Lebensqualitätsvorstellungen im Vergleich. Eine Zusammenarbeit indischer und österreichischer Jugendlicher. In: GW-Unterricht 142, 80-90.
Elbow, P. (1998): Writing with Power. Techniques for Mastering the Writing Process. 2nd ed., Oxford University Press.

EuroMemorandum (2016): Adressing Europe's multiple crises: An agenda for economic transformation, solidarity and democracy. EuroMemo Group.

Geisberger, T. (2013): Ausmaß und Struktur der Niedriglohnbeschäftigung in Österreich 2010. In: Statistik Austria (Hrsg.): Statistische Nachrichten 7. https://www. bmbf.gv.at/frauen/gender/gig_va3_artikel_niedriglohn. pdf?52h2t8 (25.04.2016).

Haug, F. \& U. Gschwandtner (2006): Sternschnuppen. Zukunftserwartungen von Schuljugend. Hamburg.

Hedtke, R. (2015): Sozioökonomische Bildung als Innovation durch Tradition. In: GW-Unterricht 140, 18-38.

OECD - Organisation for Economic Co-operation and Development (2015): In it together: Why less inequality benefits all. Paris.

Riesenfelder, A. \& P. Wetzel (2010): Die Leiharbeit in der Krise? L\&R Sozialforschung, Wien. http://www. lrsocialresearch.at/files/ENDBERICHT_AKUe.pdf (10.11.2016).

Scarpetta, S., S. Cazes \& A. Garnero (2016): Measuring the quality of jobs in OECD countries, VOX. http:// voxeu.org/article/measuring-quality-jobs-oecd-countries (21.07.2016).

Schindler, R. \& U. Schönbauer (2013): Leiharbeit aus Sicht der BetriebsrätInnen. Arbeiterkammer Wien.

Statistik Austria (2016): Armut und soziale Eingliederung 2015. Ergebnisse aus EU-SILC 2015. Präsentation zur Pressekonferenz.

Schulze Buschoff, K. (2016): Atypische Beschäftigung in Europa. Hans-Böckler Stiftung Study Nr. 1.

Teitzer, R. (2014): Atypische Beschäftigung vor und in der Krise. Working Papers on Employment and Flexibilization. Universität Wien.

Vielhaber, C. (2001): Politische Bildung in der Schulgeographie. In: Sitte, W. \& H. Wohlschlägl (Hrsg.): Beiträge zur Didaktik des „Geographie- und Wirtschaftskunde“Unterrichts (=Materialien zur Geographie und Wirtschaftskunde 16), 333-355.

Voss, E. \& K. Vitols (2013): Leiharbeit in Europa: zwischen Flexibilität und Sicherheit. In: WSI-Mitteilungen 8, 580-587.

Weinkopf, C., B. Hieming \& L. Mesaros (2009): Prekäre Beschäftigung. Expertise für die SPD-Landtagsfraktion NRW. Duisburg. 


\section{Arbeitsblatt 1: Glücksbaum}

Was erscheint Dir für Dein persönliches Wohlbefinden als besonders wichtig? Erstelle zu diesem Thema auf einem A3 Blatt deinen eigenen „Glücksbaum“. Die Aspekte, die du für dein persönliches Wohlbefinden als wichtig erachtest, bilden die Blätter dieses Baumes. Achte darauf, dass dein Baum über ausreichend viele Blätter verfügt, damit auch alle Deine Wohlfühlfaktoren abgebildet werden können!

Konzentriere Dich auf die persönlich wichtigen Aspekte Deines Wohlbefindens, ohne einen Blick auf die Aspekte deines Banknachbarn/deiner Banknachbarin zu werfen.

Ordne auf deiner Darstellung die Blätter des Baumes so an, dass bei jedem Blatt noch etwas Platz für weitere Einträge frei bleibt, und trage dann in jeweils ein Blatt einen von Dir als wichtig erachteten Wohlfühlfaktor ein. Verwende dazu einen blauen oder schwarzen Stift.

Wenn du fertig bist, vergleiche Deinen persönlichen Glücksbaum mit jenem deines Banknachbarn/deiner Banknachbarin. Welche Aspekte decken sich, wo liegen die Unterschiede? Diskutiert darüber und listet Gemeinsamkeiten und Unterschiede auf.

\section{Arbeitsblatt 2: Gefährdetes Glück}

Äußere Einflüsse können dazu führen, dass Aspekte, die wir für unser Wohlbefinden als wichtig erachten, plötzlich gefährdet sind.

Überlege dir nun in Einzelarbeit für jeden deiner auf dem Baum ausgewiesenen Wohlfühlfaktoren mindestens ein konkretes Ereignis, durch das der jeweilige Wohlfühlfaktor gefährdet sein könnte. Ergänze nun die einzelnen Blätter deines Baumes um diese „Störfaktoren“, die dein persönliches Wohlbefinden negativ beeinflussen könnten. Verwende dazu einen roten Stift.

Vergleiche nun deinen persönlicher Glücksbaum mit jenem deines Banknachbarn/deiner Banknachbarin. Welche Aspekte decken sich, wo liegen die Unterschiede? Diskutiert darüber und listet Gemeinsamkeiten und Unterschiede auf. 


\section{Material 1}

Wie wirkt sich der Wechsel von Normalarbeit zu prekärer Arbeit auf das Leben einer Familie aus? Die folgende erfundene Geschichte stellt eine solche Veränderung dar. Es handelt sich dabei um eine Familie mit zwei Kindern (Mutter 47 Jahre, Vater 50 Jahre, Tochter 17 Jahre, Sohn 15 Jahre). Vergleiche das neue, geringere Familieneinkommen auch mit den Angaben im Material 2.

\begin{tabular}{|c|c|}
\hline $\begin{array}{l}\text { VORHER } \\
\text { Familienleben mit Normalarbeitsverhältnis }\end{array}$ & $\begin{array}{l}\text { NACHHER } \\
\text { Familienleben mit prekärem Arbeitsverhältnis }\end{array}$ \\
\hline $\begin{array}{l}\text { - Wohnort: Umland von Wien, } 100 \mathrm{~m}^{2} \text { Mietwohnung } \\
\text { - Die Familie besitzt ein Auto, mit dem der Vater täglich } \\
\text { in seine Arbeit nach Wien fährt. Dabei nimmt er } \\
\text { auch seine Tochter mit, die in Wien eine HTL besucht. } \\
\text { Der Sohn besucht ein Gymnasium, das er mit dem } \\
\text { Schulbus erreicht. } \\
\text { Der Vater ist Facharbeiter in einer Großputzerei } \\
\text { und verdient netto } € 2000 \text {, während die Mutter im } \\
\text { Wohnumfeld im Verkauf arbeitet und netto } € 1400 \\
\text { verdient. Sie kann ihre Arbeit mit dem Rad erreichen. }\end{array}$ & $\begin{array}{l}\text { Der Vater verliert seine Arbeit und findet nach sie- } \\
\text { ben Monaten Arbeitslosigkeit einen neuen Job als } \\
\text { Leiharbeiter. Er ist aber nicht mehr Vollzeitbeschäftigt } \\
\text { sondern arbeitet nur mehr im Ausmaß von ca. } 70 \% \\
\text { des Normalarbeitsverhältnisses ( } 27 \text { statt 38,5 Stun- } \\
\text { den pro Woche), weil er nicht für eine längere Zeit } \\
\text { gebraucht wird. } \\
\text { Der Lohn des Vaters beträgt nunmehr netto ca. } \\
€ 1000, \text { wobei er zusätzlich noch ca. alle vier Mo- } \\
\text { nate an ein anderes Unternehmen ausgeliehen wird. } \\
\text { Zumeist handelt es sich dabei um die folgenden drei } \\
\text { Branchen: Gastronomie, Wachdienste, Reinigungs- } \\
\text { personal. Seine Aussichten auf eine fixe Beschäfti- } \\
\text { gung auf Basis seiner Qualifikationen als Facharbei- } \\
\text { ter sind schlecht. }\end{array}$ \\
\hline
\end{tabular}

\section{Material 2}

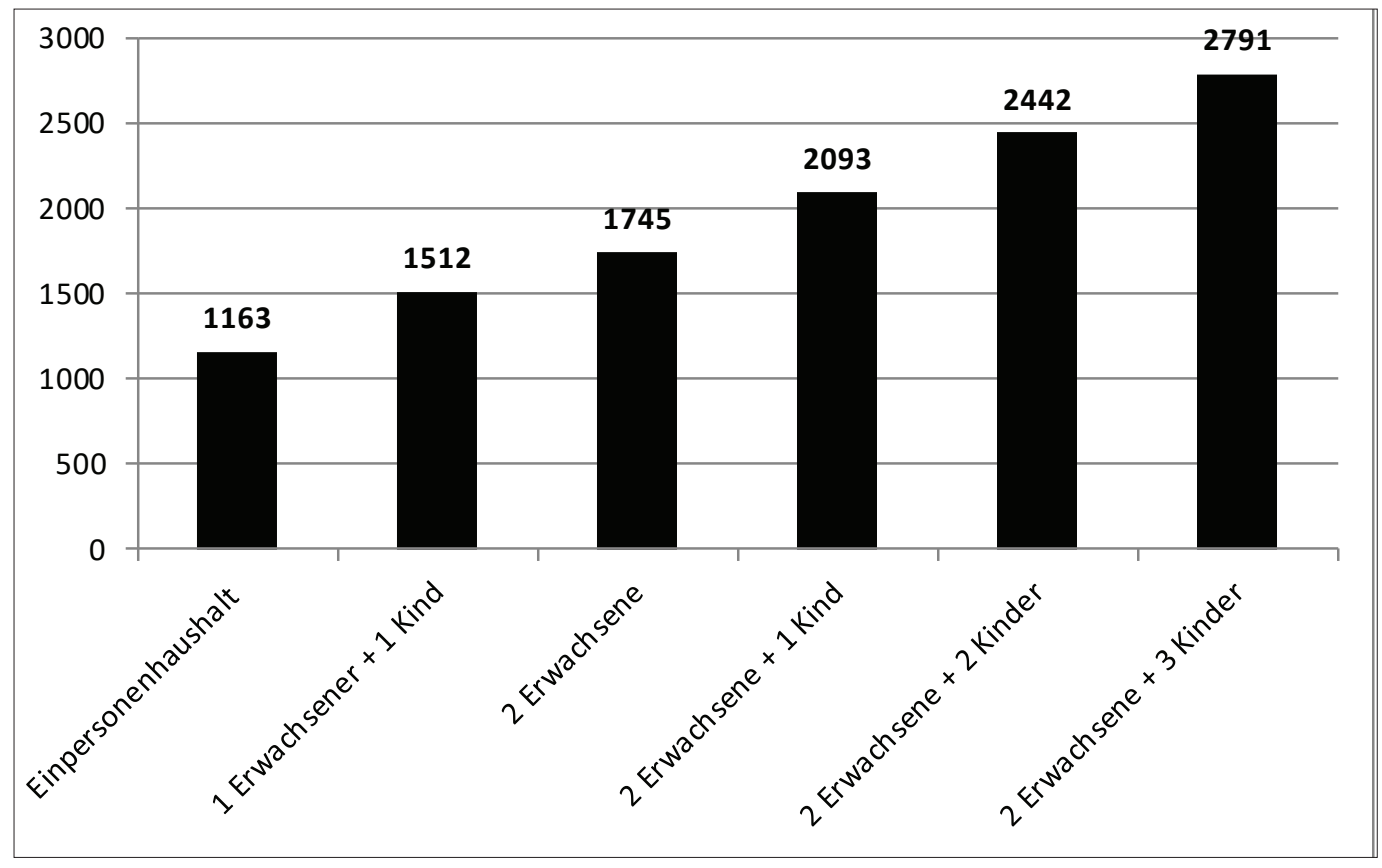

Armutsgefährdungsschwellen für unterschiedliche Haushaltstypen in Österreich für das Jahr 2015, Monatswerte in Euro (eigene Darstellung nach Daten aus Statistik Austria 2016) 
Arbeitsblatt 3: Haushaltsausgaben bei Normalarbeitsverhältnis

Budget der Familie: $€ 3400$

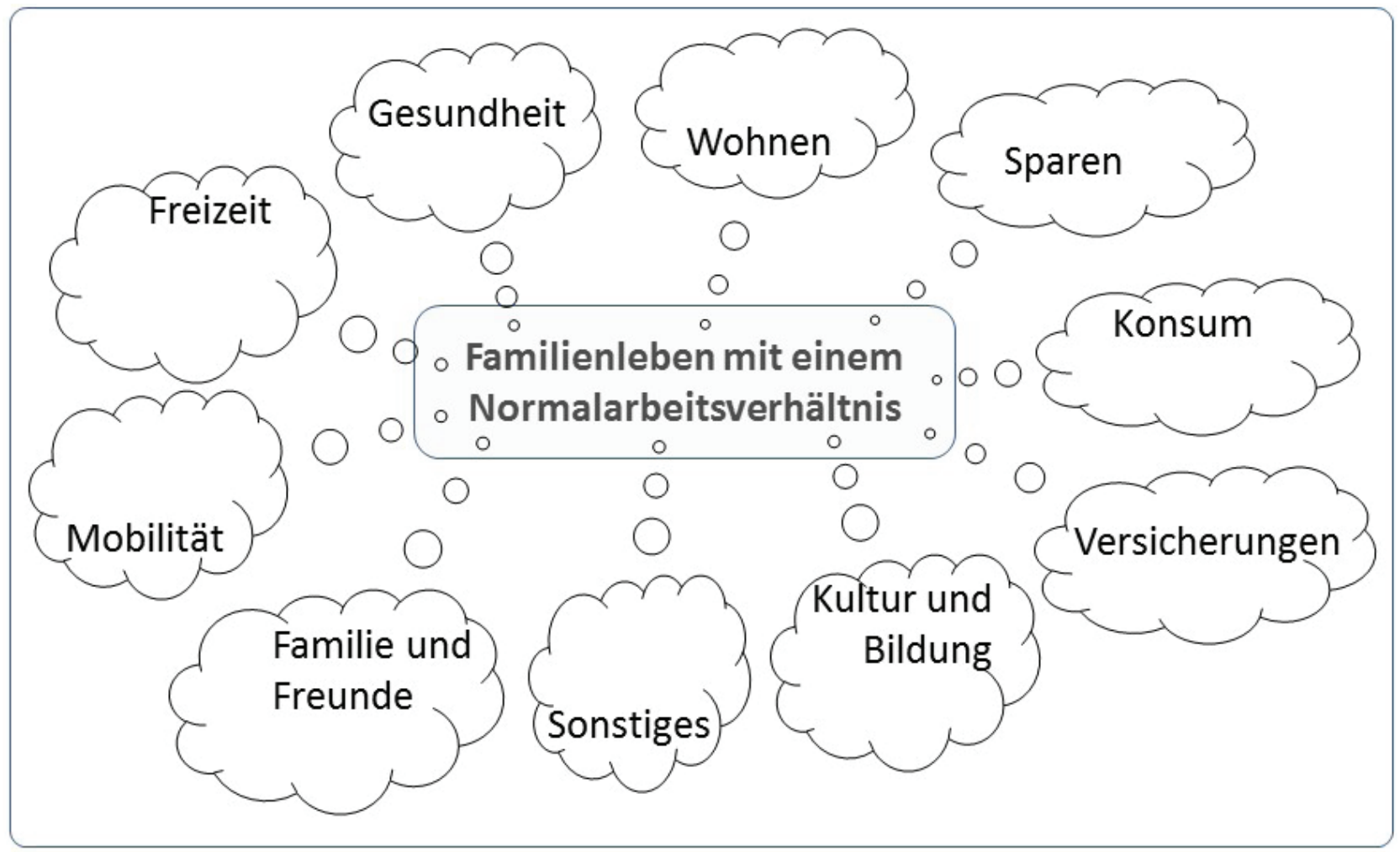

Arbeitsblatt 4: Haushaltsausgaben bei prekärem Beschäftigungsverhältnis

Budget der Familie: $€ 2400$

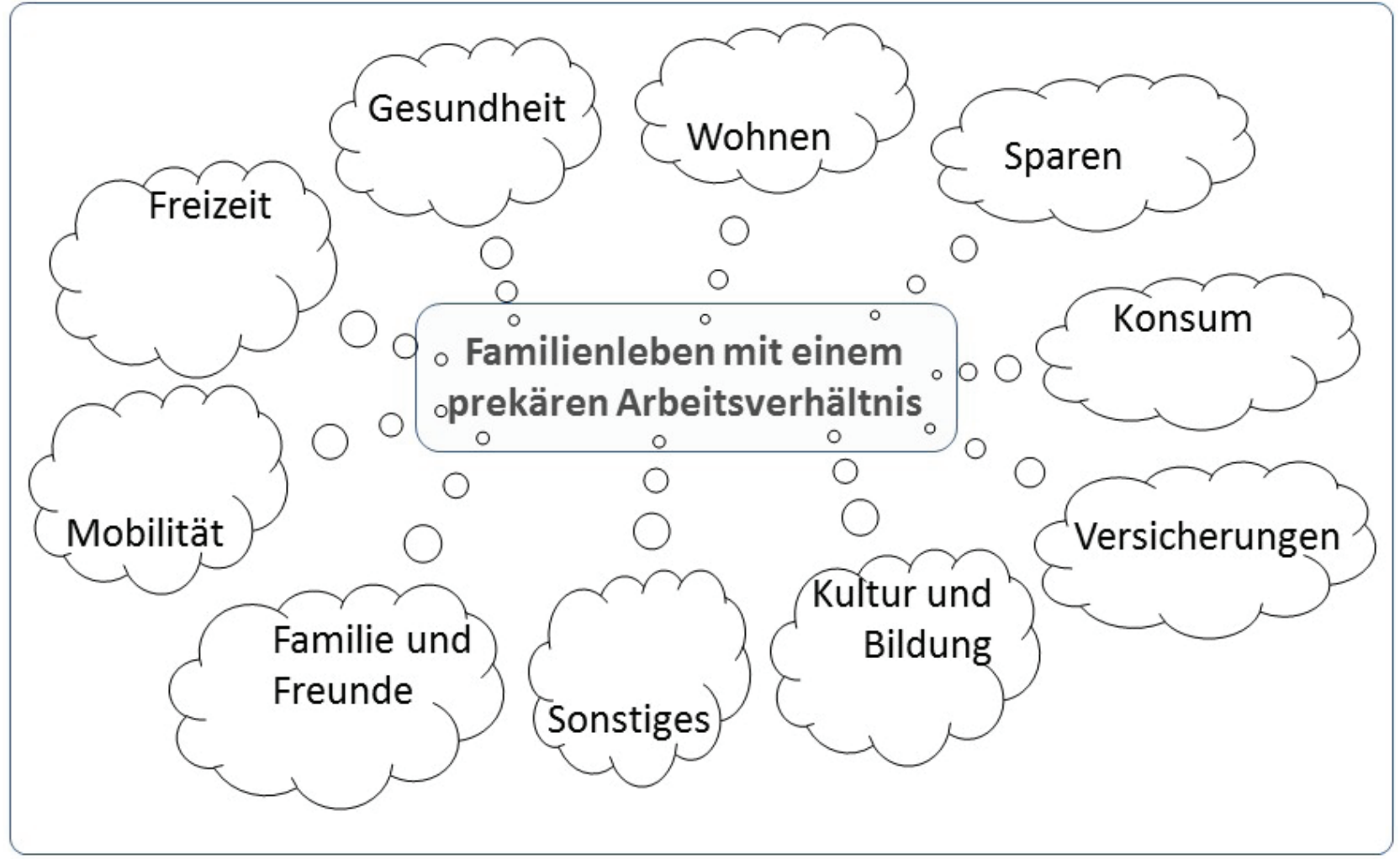

